\title{
Correspondence between many-particle excitations and the entan- glement spectrum of disordered ballistic one-dimensional systems
}

\author{
Shaul Leiman ${ }^{1}$, Ariel Eisenbach ${ }^{1}$ and Richard Berkovits ${ }^{1}$ \\ ${ }^{1}$ Department of Physics, Jack and Pearl Resnick Institute, Bar-Ilan University, Ramat-Gan 52900, Israel \\ PACS 61.43.-j-Disordered Solids \\ PACS 73.21. Hb - Quantum Wires \\ PACS 03.65.Ud - Entanglement and Quantum Non-locality
}

\begin{abstract}
Using exact diagonalization for non-interacting systems and density matrix renormalization group for interacting systems we show that Li and Haldane's conjecture on the correspondence between the low-lying many-particle excitation spectrum and the entanglement spectrum holds for disordered ballistic one-dimensional many-particle systems. In order to demonstrate the correspondence we develope a computational efficient way to calculate the ES of low-excitation of non-interacting systems. We observe and explain the presence of an unexpected shell structure in the excitation structure. The low-lying shell are robust and survive even for strong electronelectron interactions.
\end{abstract}

Introduction. - Concepts developed in the field of quantum information are gaining a foothold in condensed mater physics [1,2]. One of the most influential concepts 'is related to quantifying the entanglement between two parts of a system. Specifically, a many particle system in ' a pure state, may be divided into two regions $\mathrm{A}$ and $\mathrm{B}$. The entanglement between the regions $\mathrm{A}$ and $\mathrm{B}$ can be ' quantified by different measures such as the entanglement entropy, Rényi entropy and entanglement spectrum (ES), connected to the reduced density matrix of area $\mathrm{A}, \rho_{A}$ or $\mathrm{B}, \rho_{B}$

The canonical method for utilizing the information embedded in the eigenvalues $\lambda_{i}^{A}$ of $\rho_{A}$ is the entanglement 'entropy: $S_{A}=-\sum_{i} \lambda_{i}^{A} \ln \lambda_{i}^{A}$. The ES is constructed out of the set of eigenvalues $\left\{\lambda_{i}^{A}\right\}$ by the transformation: $\left\{\varepsilon_{i}^{A}=-\ln \lambda_{i}^{A}\right\}$. Several years ago Li and Haldane [3] came up with an intriguing conjecture regarding the ES of a many-body state. They show that the ES of a partitioned fractional quantum Hall $\nu=5 / 2$ state resembled the minimal edge excitation spectrum, and suggest a connection between the properties of the ES and the topological order of this state.

This idea led to many additional studies [4 21], mainly focused on systems with topological behavior, suggesting that the low-energy ES distribution shows some correspondence to the true many-body excitations (MBE) of the partitioned segment (region A). Since the reduced density matrix of a region is adiabatically connected to the $\mathrm{MBE}$ of the disconnected region, one may expect that the exact ground state eigenfunction of the whole system encodes information on the sub-system's low lying excitations.

In this letter we would use Li and Haldanes' conjecture in order to investigate properties of the excitation spectrum of fermionic disordered systems. The effect of disorder on the ES of edge states in topological insulators has been previously studied 22,23. Properties of several low lying ES levels have been used to identify a metallic phase in one-dimensional Bose-Hubbard models 24. Nevertheless, a direct comparison between the properties of many particle excitations in a weakly disordered systems and the ES of a corresponding segment is still lacking.

The field of MBE statistics has a long history, and continues to draw interest in diverse areas such as cold atoms in the presence of quasi-periodic potentials $25-28$, and the many body localization (MBL) transition 29 31. For disordered systems it is natural to seek knowledge on the statistical properties of spectrum. Specifically, we are interested in answering the question: Does the ES exhibit statistical properties corresponding to the MBE spectrum. For the disordered single particle spectrum there is an extensive literature on the statistical properties of the energy spectrum in the localized, critical, diffusive and chaotic regimes. Different energy spectrum and wave function statistics, depending on whether the disordered system has time reversal symmetry (Gaussian orthogonal ensemble (GOE)), broken time reversal symmetry (Gaussian uni- 
tary statistics (GUE)), spin-orbit interactions (Gaussian symplectic statistics (GSE)) 32 34. The energy spectrum statistics can be used to identify the Anderson localization transition [35.

The statistics of MBE in disordered interacting systems have an interesting twist. For non-interacting manyparticle systems the level spacing MBE distribution is expected to follow the Poisson distribution for excitation energies above the second spacing, without depending on the single-level distribution [36. On the other hand, once repulsive interactions between the particles are considered, a transition to the Wigner distribution for higher excitations is observed [37 41]. The main difficulty in studying this transition is that exact diagonalization needed to study excited states is limited to very small systems. Here, the $\mathrm{Li}$ and Haldanes' conjecture can come to the rescue, since as we shall demonstrate below, one may extract the ES up to a few hundreds of states. Thus, if indeed there is a connection between the ES and The MBE, the low level excitations of a rather large many-body systems are numerically available.

In this letter we shall explicitly demonstrate the correspondence between the excitation spectrum and the ES, for non-interacting one-dimensional disordered systems in the chaotic regime. In order to facilitate the calculation of the ES for large systems, we present a numerical method based on the correlation matrix eigenvalues, which could be used for non interacting systems in any dimension. We show that the average entanglement spectrum level spacings (ESLS) show a shell structure, with a large average spacing appearing according to the combinatorical partition function $p(m)$. The ESLS follows the Poisson statistics for the small spacings, while it shows a narrow distribution for the large spacings. Adding interactions removes the shell structure for strong interactions at higher levels, but the shell structure is rather robust for the low-lying portion of the ES, corresponding to the MBE which are close to the Fermi energy.

Model. - As an example of a disordered manyparticle system, we consider a spinless $1 \mathrm{D}$ electrons system of size $L$ with repulsive nearest-neighbor interactions and on-site disordered potential. The Hamiltonian is given by:

$$
\begin{aligned}
H & =\sum_{j=1}^{L} \epsilon_{j} \hat{c}_{j}^{\dagger} \hat{c}_{j}-t \sum_{j=1}^{L-1}\left(\hat{c}_{j}^{\dagger} \hat{c}_{j+1}+\text { h.c. }\right) \\
& +U \sum_{j=1}^{L-1}\left(\hat{c}_{j}^{\dagger} \hat{c}_{j}-\frac{1}{2}\right)\left(\hat{c}_{j+1}^{\dagger} \hat{c}_{j+1}-\frac{1}{2}\right),
\end{aligned}
$$

where $\epsilon_{j}$ is the on-site energy drawn from a uniform distribution $[-W / 2, W / 2], \hat{c}_{j}^{\dagger}$ is the creation operator of an electron at site $j$, and $t=1$ is the hopping matrix element. The repulsive interaction strength is depicted by $U \geq 0$, and a positive background is considered.

Non interacting electrons. - All single-electron states of such a $1 \mathrm{D}$ system are localized [43, with states

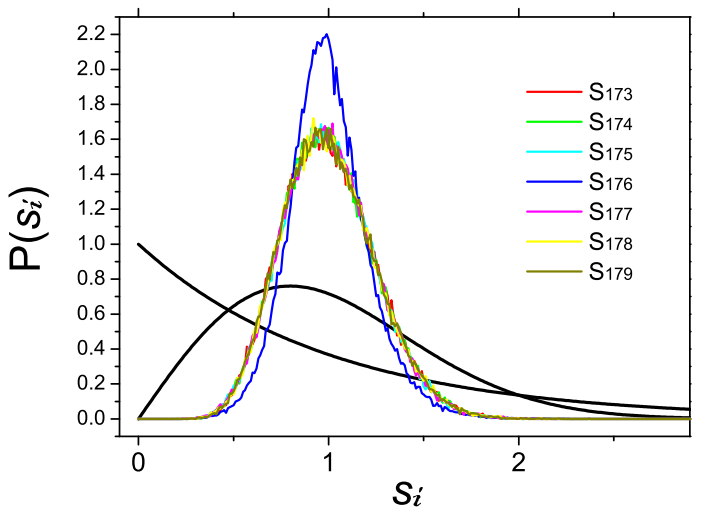

Fig. 1: The single electron level spacing distribution $P\left(s_{i}\right)$ for states $i=173 \ldots 179$ around the middle of the band for a $L=350, W=0.3$ wire. For this strength of disorder the system is disordered chaotic $L<\xi$, and the distribution is peaked around the mean (where the level at the middle of the band $i=176$ is more peaked than the others). The Poisson and GOE distributions are provided for comparison.

at the middle of the band having a localization length $\xi \approx 105 / W^{2}$ [44. We consider a case where the disorder was chosen as $W=0.3$ and $L=350$, i.e., $\xi \sim 1100>L$. For this case the single electron energies $\varepsilon_{i}$ an eigenstates $\psi_{i}$ are readily available via exact diagonalization. Unless otherwise specified, we perform our analysis over 10, 000 realizations of disorder. First we consider the single electron level-spacing distribution, for the $i$-th spacing $\delta_{i}=\varepsilon_{i+1}-\varepsilon_{i}$. The unfolded spacing is defined as $s_{i}=\delta_{i} /\left\langle\delta_{i}\right\rangle$, where $\langle\ldots\rangle$ denotes averaging over the different disorder realization. The single electron level-spacing will follow the Poisson statistics $\left(P_{\text {Poisson }}\left(s_{i}\right)=\exp \left(-s_{i}\right)\right)$ as long as $L \gg \xi$, while in the metallic (diffusive) regime it should follow the Wigner $(\mathrm{GOE})$ distribution $\left(P_{\mathrm{GOE}}\left(s_{i}\right)=\right.$ $\left.\left(\pi s_{i} / 2\right) \exp \left(-\pi s_{i}^{2} / 4\right)\right)$ [35. For a one-dimensional disordered system there is no genuine metallic regime since $L<\xi \sim \ell$ (where $\ell$ is the mean free path) and therefore the system crosses from a localized to a disordered ballistic regime, resulting in a narrower distribution concentrated around the mean $\left(s_{i}=1\right)$. This can be clearly seen in Fig. 1, where the distribution of the single electron level spacings close to the middle of the band of a ballistic system is presented.

The MBE of the system can not be obtained by exact diagonaliztion since the size of the many-body Hilbert space grows as $\left(\begin{array}{c}L \\ N\end{array}\right)$, which for half-filling $(N=L / 2=175)$, where $N$ is the number of particles, is of order $10^{104}$. Thus, another tack is needed. For the non-interacting case one may calculate the low-lying excitations by considering the different permutations of the single electron occupations, $n_{i}=0,1$, of the single electron states. Here, each permutation which obeys $N=\sum_{i} n_{i}$ is a many-body state, with 

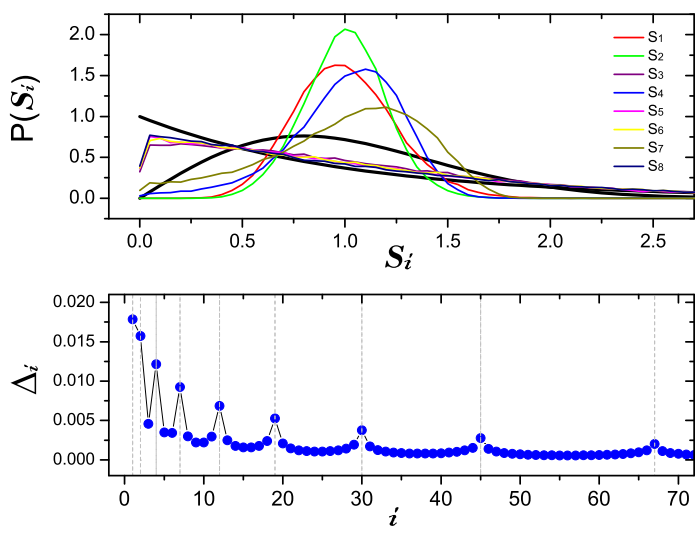

Fig. 2: The properties of the many-body excitation spectrum for 10000 realizations of the non-interacting system $(U=0)$ whose single electron level spacings is depicted in Fig. 11 Top panel: Level spacing distributions for the eight lowest manyparticle levels. Lower panels: Average spacings as function of the level number. The vertical line depict the positions of the peaks predicted by the partition function $p(n)$.

the total many body energy $E=\sum_{i} n_{i} \varepsilon_{i}$. These manybody states may be enumerates by arranging the energies, such that $E_{1}<E_{2}<E_{3}<\ldots$. Of course there are still an astronomical number of these states and covering them all is impossible. Nevertheless, if one constrains the search to low lying excitations, one can limit the the permutations to $p$ (of order $p \sim E / \delta$ ) single electron states around the Fermi energy. Thus, only $\left(\begin{array}{c}2 p \\ p\end{array}\right)$ MBE are considered. Similar to the single particle case the MBE level spacing $\Delta_{i}=E_{i+1}-E_{i}$ can be extracted, as well as the average $\left\langle\Delta_{i}\right\rangle$ and distribution $P\left(S_{i}\right)$, where $S_{i}=\Delta_{i} /\left\langle\Delta_{i}\right\rangle$.

The results for the average MBE spacings and their distribution are plotted on the right panels of Fig. 2 . It is immediate apparent that the average MBE spacing exhibits a shell-like (also known as magic numbers in context of nuclear physics) structure. Spacings $1,2,4,7,12,19,30,45,67 \ldots$ are significantly larger than their neighboring spacings. The reason for this behavior is combinatorical. The large spacing corresponds to an excitation different from the previous one by a single electron moving to the next available single-electron state. On the other hand the typical smaller spacings corresponds to the movement of several electrons between different single-electron states. For equal singlelevel spacings (i.e., $\delta_{i}=\delta$ ), the MBE can acquire energies of $m \delta$ above the ground state where $m=1,2, \ldots$. The degeneracy of the states with energy $m \delta$ depends on the the number of ways of writing the integer $m$ as a sum of positive integers, where the order of addends is not significant. This corresponds to a problem in combinatorics knows as the partition problem, and the answer is given by the partition function $p(m)$ (which
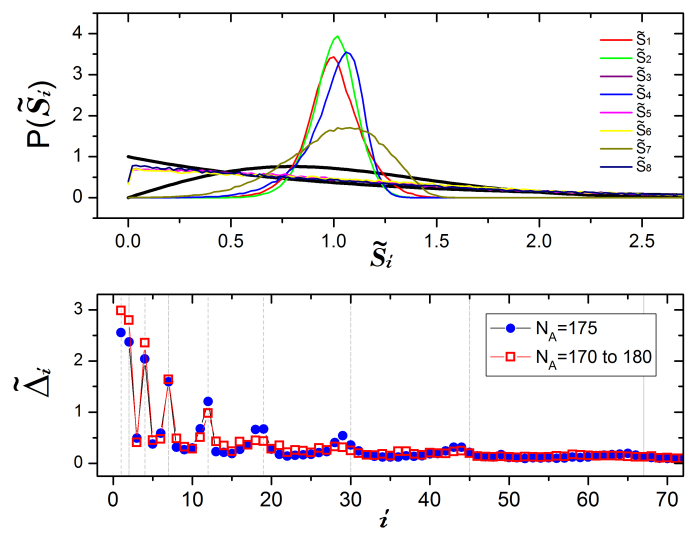

Fig. 3: The properties of the many body entanglement spectrum for an $L_{A}=350$ region out of a $L=700$ system, with $U=0$, averaged over 10000 realizations of disorder. Top panel: Entanglement level spacing distributions for the eight lowest levels for $N_{A}=L_{A} / 2$. Lower panel: Average spacings as function of the entanglement level number. Blue circles correspond to $N_{A}=L_{A} / 2$. Red squares to an additional average over $L_{A} / 2-6<N_{A}<L_{A} / 2+6$.

is not the thermodynamical partition function), which has a recurrence relation $p(m)=\sum_{k=1}^{m}(-1)^{k+1}[p(m-$ $k(3 k-1) / 2)+p(m-k(3 k+1) / 2)$ ] [45]. Thus, $p(m)$ for $m=1,2, \ldots$ are $1,2,3,5,7,11,15,22,30,42, \ldots$ Hardy and Ramanujan have devised an asymptotic form $p(m) \sim$ $(4 m \sqrt{3})^{-1} \exp (\pi \sqrt{2 m / 3})$ [46. In the case where the single level spacing is not constant the degeneracy is lifted, but as long as the variation is not too large, the shell structure remains and the MBE level spacing between a closed shell and the next open one is relatively large, as can be seen in Fig. 2. Thus, the number of MBE states between the $j$-th MBE shell (i.e., large level spacing) and $j+1$ shell is $p(j)$, in agreement with the numerical results.

The MBE spacing distributions $P\left(S_{i}\right)$ also retain the shell structure, where the distributions corresponding to the large spacings are similar to the single electron spacings (Fig. 1), i.e., narrow and peaked around the mean, while for the other spacings follow the Poisson distribution, expected when the non-interacting MBE differ by more than a single electron occupation [36, 37] (see Fig. 2).

Does the ES of a finite segment of a disordered system exhibit similar behavior as might be expected from the $\mathrm{Li}$ and Haldanes' conjecture [3] F First we have to calculate the ES, i.e., the eigenvalues of the RDM. The density matrix renormalization group (DMRG) [47,48, is a natural candidate for calculating the ground state of disordered interacting 1D system and the corresponding eigenvalues of the reduced density matrix. We will use it for the interacting case. For non-interacting systems, one would expect that it is possible to extract the eigenvalues without to resort to DMRG. Direct calculation of the RDM is 
impossible since its size is of order $2^{L_{A}}$ (where $L_{A}$ is the size of region A), so a different approach is needed.

Reduced density matrix eigenvalues using the correlation matrix. - In order to calculate the ESLS for the non-interacting case we use the connection between the correlation matrix $(\mathrm{CM})$ and the RDM [42. The CM between two sites in region $\mathrm{A}$ is a unitary matrix given by:

$$
C_{m n}^{\dagger}=\left[\operatorname{Tr}\left\{\rho_{A} c_{m}^{\dagger} c_{n}\right\}\right]^{\dagger}=\operatorname{Tr}\left\{c_{n}^{\dagger} c_{m} \rho_{A}\right\}^{=} C_{n m} .
$$

One may diagonalize the $\mathrm{CM}$ and write the trace in terms of the eigenvectors. Thus,

$$
\operatorname{Tr}\left\{\rho_{A} a_{q}^{\dagger} a_{p}\right\}=\nu_{q} \delta_{q p}
$$

where $a_{q}^{\dagger}$ is the creation operator of the $q$-th eigenvector and $\nu_{q}$ is the corresponding eigenvalue. The RDM for noninteracting electrons can be written as an product state of local density matrices [42]:

$$
\rho_{A}=\rho_{1} \otimes \rho_{2} \otimes \ldots \otimes \rho_{L_{A}}
$$

where $\rho_{q}$ is the density matrix associated with the $q^{\prime}$ th eigenvalue of the CM. Moreover, due to the fermionic nature of the particles, $\rho_{q}$ has to be diagonal.

The relation between $\nu_{q}$ and $\rho_{q}$ is easily extracted [42]:

$$
\begin{array}{r}
A_{q q}=\operatorname{Tr}\left\{\rho_{A} a_{q}^{\dagger} a_{q}\right\}=\operatorname{Tr}\left\{\rho_{q} a_{q}^{\dagger} a_{q}\right\}= \\
=\operatorname{Tr}\left\{\left(\begin{array}{cc}
1-\nu_{q} & 0 \\
0 & \nu_{q}
\end{array}\right)\left(\begin{array}{cc}
0 & 0 \\
0 & 1
\end{array}\right)\right\}=\nu_{q} .
\end{array}
$$

Since $\rho_{q}$ are independent any eigenvalue of the RDM can be constructed by multiplying a permutation of either $\left(1-\nu_{q}\right)$ (no particle) or $\nu_{q}$ (one particle) occupying the $q$-th state. For the case where no particles occupy region $\mathrm{A}\left(N_{A}=0\right)$, the single eigenvalue of the $\mathrm{RDM}$ is $\lambda_{1}^{N_{A}=0}=\prod_{q=1}^{L_{A}}\left(1-\nu_{q}\right)$. For the $N_{A}=1$ the RDM has $L_{A}$ eigenvalues, where the $i$-th eigenvalue, $\lambda_{i}^{N_{A}=1}=\left(1-\nu_{1}\right)\left(1-\nu_{2}\right) \ldots \nu_{i} \ldots\left(1-\nu_{L_{A}}\right)$. Generalizing to any $N_{A}=p$ particle block in the RDM the first eigenvalue is constructed by $\lambda_{1}^{p}=\nu_{1} \ldots \nu_{p}\left(1-\nu_{p+1}\right)\left(1-\nu_{L_{A}}\right)$, while the other $\left(\begin{array}{c}L_{A} \\ p\end{array}\right)$ permutations define the rest of the eigenvalues. Since usually many of the CM eigenvalues are either extremely small or very close to one, it is possible to reach an accurate estimation of $\lambda$, with significantly fewer permutations.

The ES obtained for a wire of length $L=700$ and $L_{A}=350$ occupied by $N_{A}$ particles and averaged over 10,000 realizations of disorder. are presented in Fig. 3 The occupation is $N_{A}=L_{A} / 2$ or averaged for values of $L_{A} / 2-6<N_{A}<L_{A} / 2+6$. This size was chosen in order to correspond to the sizes of the system for which the MBE were calculated. For the ES we perform the transformation $\epsilon_{i}^{N_{A}}=-\ln \left(\lambda_{i}^{N_{A}}\right)$ and calculate the ESLS $\tilde{\Delta}_{i}^{N_{A}}=\epsilon_{i+1}^{N_{A}}-\epsilon_{i}^{N_{A}}$, the average spacing $\left\langle\tilde{\Delta}_{i}\right\rangle$ and distribution $P\left(\tilde{S}_{i}\right)$, where $\tilde{S}_{i}=\tilde{\Delta}_{i} /\left\langle\tilde{\Delta}_{i}\right\rangle$. Indeed, the low-lying
ESLS average as well as the spacing distributions show the same general features shown by the MBE. The shell structure is reproduced for spacings $1,2,4,7,12$, small deviations in the peak positions appear for the higher spacings at 19 and 30, and is almost wiped up at peaks higher than 45. The general behavior of the ESLS follows the distribution exhibited by the excitation spacings with narrow distributions for spacings 1,2,4 and 7, while the other spacings follow the Poisson distribution. Nevertheless, details such as the exact width of the distribution vary between the excitation and entanglement spacings.

The influence of interactions. - Once interactions are added, our previous arguments do not hold anymore. Nevertheless, following the ideas leading to the Fermi liquid picture and quasi particles, one expects that for not too strong interactions and close to the Fermi energy the non-interacting shell picture will continue to provide a good description of the system.

As we can not use the combinatoric approach to calculate the interacting MBE energy levels, and neither exact diagonaliztion nor DMRG can provide more than a few excited states, we will use the correspondence between the MBE and the ES of a finite section shown for the noninteracting case to study the evolution of the spectrum as function of electron-electron interaction strength. Thus we turn on the interaction $U$ in Eq. (11) and use DMRG to calculate the ES over 100 realizations of the same disorder, wire length, size of region A, and number of particles as for the non-interacting case. The average ESLS $\tilde{\Delta}_{i}$, and distribution $P\left(\tilde{S}_{i}\right)$, are calculated and presented in Fig. 4. For weak interactions $U=0.3$, the shell structure of the average spacing is hardly affected. As interactions increase, the higher shells are washed out, until for $U=2.4$ only spacings 1,2 and 4 remain larger than their neighbors. The entanglement spacing distribution is also transformed as function of interaction strength. For weak interactions $(U=0.3)$ the distribution $P\left(\tilde{S}_{3}\right)$ is close to Poisson, while the distribution for the 4 -th (large) spacings, $P\left(\tilde{S}_{4}\right)$, is peaked around the mean, as for the non-interacting case (see inset Fig. (4). This distinction is blurred as the interactions become stronger and $P\left(\tilde{S}_{i}\right)$ for any $i$ approaches the GOE distribution. This is in agreement with the observations for the distribution of interacting MBE [36 41$]$ which show a transition to GOE statistics as interactions become stronger. Thus, it seems that the Li and Haldane conjecture holds even for the ES of interacting systems.

Discussion. - Thus, Li and Haldanes' conjecture on the correspondence between the low-lying MBE spectrum, and its ES is confirmed for non-interacting disordered systems. Turning on interactions gradually change the ES of the system in a manner that fits the expectations for the behavior of the MBE. We conclude that ES is a promising route to study MBE properties beyond the first few excitations, which are impossible to study by any other way for large systems. This could be a promising way to study phenomena occurring only for the MBE, such 


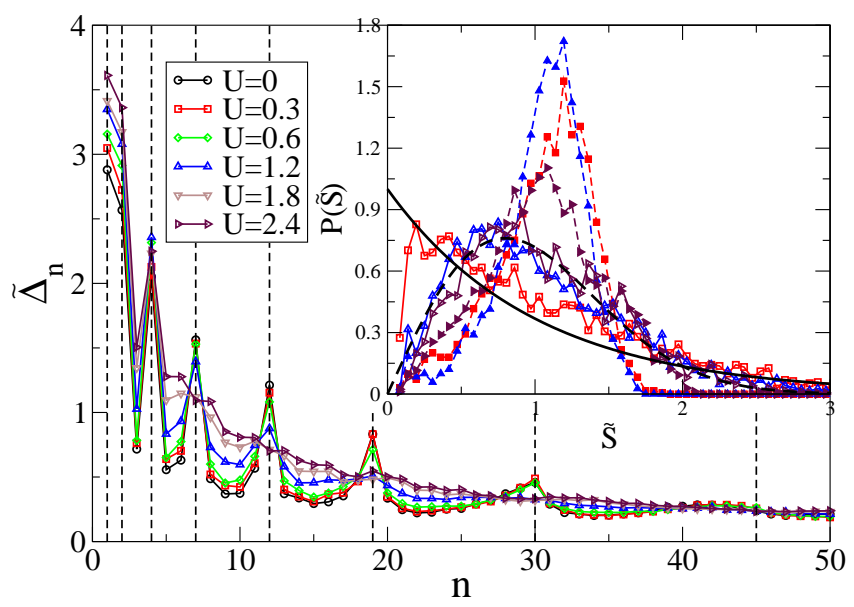

Fig. 4: Averaged entanglement spectrum level spacings $\tilde{\Delta}_{n}$ as function of the level number $n$ for different interaction strength $U$. Inset: The spacing, $\tilde{S}$, distribution for the 3 rd (continuous line, empty symbols) and 4th (dashed line, full symbols) for $U=0.3,1.2$ and 2.4. The Poisson (continuous black line) and GOE (dashed black line) are drawn for comparison.

as the many-body localization transition 29. Although still limited to low-lying excitations, the fact that higher many-body excitation may be probed improves the possibility to glean useful information on the transition. Indeed in the behavior of the shell structure as function of interaction strength presented in Fig. 4 where as interaction increases lower shell peaks disappear, may show a glimpse of this phenomena.

For non-interacting systems we have presented a computational effective method to extract the low-lying ES from the CM. This method could be used for the study of the ES for higher dimension, which is otherwise quite daunting.

We have also shown a new shell (magic number) structure appearing for disordered ballistic one dimensional systems and explained its origin. The low-lying shells are robust and are not wiped out by interactions. It is interesting to understand whether this behavior survives for stronger disorder where the localization length is smaller than the length of region A. On one hand, since the disorder is larger it is expected to wipe out the shell structure, on the other hand, the area in region A entangled with the rest of the system remains of order of $\xi$ and thus shrinks with the disorder, i.e., one effectively samples a smaller sample. Also the study of higher dimensions may prove interesting.

We would like to thank B. L. Altshuler for useful discussions and the Institute of Basic Science Center for Theoretical Physics of Complex Systems, Daejeon, South Korea where part of this work was performed.

\section{REFERENCES}

[1] L. Amico, R. Fazio, A. Osterloh, and V. Vedral, Rev. Mod. Phys. 80, 517 (2008).

[2] J. Eisert, M. Cramer, and M. B. Plenio, Rev. Mod. Phys. 82, 277 (2010).

[3] H. Li and F. D. M. Haldane, Phys. Rev. Lett. 101, 010504 (2008).

[4] P. Calabrese and A. Lefevre, Phys. Rev. A 78, 032329 (2008).

[5] A. M. Läuchli, E. J. Bergholtz, J. Suorsa, and M. Haque, Phys. Rev. Lett. 104, 156404 (2010).

[6] H. Yao and X.-L. Qi, Phys. Rev. Lett. 105, 080501 (2010).

[7] R. Thomale, A. Sterdyniak, N. Regnault, and B. A. Bernevig, Phys. Rev. Lett. 104, 180502 (2010).

[8] L. Fidkowski, Phys. Rev. Lett. 104, 130502 (2010).

[9] F. Pollmann, A. M. Turner, E. Berg, and M. Oshikawa, Phys. Rev. B 81, 064439 (2010).

[10] D. Poilblanc, Phys. Rev. Lett. 105, 077202 (2010).

[11] M. Hermanns, A. Chandran, N. Regnault, and B. A. Bernevig, Phys. Rev. B 84, 121309 (2011).

[12] J. Dubail and N. Read, Phys. Rev. Lett. 107, 157001 (2011)

[13] A. Chandran, M. Hermanns, N. Regnault, and B. A. Bernevig, Phys. Rev. B 84, 205136 (2011).

[14] X.-L. Qi, H. Katsura, and A.W.W. Ludwig, Phys. Rev. Lett. 108, 196402 (2012).

[15] D. Poilblanc, N. Schuch, D. Pérez-García, and J. I. Cirac, Phys. Rev. B 86, 014404 (2012).

[16] B. Swingle and T. Senthil, Phys. Rev. B 86, 045117 (2012).

[17] J. I. Cirac, D. Poilblanc, N. Schuch, and F. Verstraete, Phys. Rev. B 83, 245134 (2011).

[18] V. Alba, M. Haque, and A. M. Läuchli, Phys. Rev. Lett. 108, 227201 (2012).

[19] V. Alba, M. Haque, and A. M. Läuchli, Phys. Rev. Lett. 110, 260403 (2013).

[20] F. Kolley, S. Depenbrock, I. P. McCulloch, U. Schollwöck, and V. Alba, Phys. Rev. B 88, 144426 (2013).

[21] A. Chandran, V. Khemani, and S. L. Sondhi, Phys. Rev. Lett. 113, 060501 (2014).

[22] E. Prodan, T. L. Hughes, and B. A. Bernevig, Phys. Rev. Lett. 105, 115501 (2010).

[23] M. J. Gilbert, B. A. Bernevig, and T. L. Hughes, Phys. Rev. B 86, 041401(R) (2012).

[24] A. M. Goldsborough and R. A. Römer, arXiv:1503.02973

[25] G. Roati, C. DErrico, L. Fallani, M. Fattori, C. Fort, M. Zaccanti, G. Modugno, M. Modugno, and M. Inguscio, Nature 453, 895 (2008).

[26] Y. Lahini, R. Pugatch, F. Pozzi, M. Sorel, R. Morandotti, N. Davidson, and Y. Silberberg, Physical Review Letters 103, 013901 (2009).

[27] L. Tanzi, E. Lucioni, S. Chaudhuri, L. Gori, A. Kumar, C. D'Errico, M. Inguscio, and G. Modugno, Phys. Rev. Lett. 111, 115301 (2013).

[28] C. D Errico, E. Lucioni, L. Tanzi, L. Gori, G. Roux, I. P. McCulloch, T. Giamarchi, M. Inguscio, and G. Modugno, Phys. Rev. Lett. 113, 095301 (2014).

[29] D. Basko, I. L. Aleiner, and B. L. Altshuler, Annals of Physics 3211126 (2006).

[30] D. Basko, I. L. Aleiner, and B. L. Altshuler, Phys. Rev. B 76, 52203 (2007). 
[31] I. V. Gornyi, A. D. Mirlin, and D. G. Polyakov, Phys. Rev. Lett. 95, 206603 (2005).

[32] F. J. Dyson and M. L. Mehta, J. Math. Phys. 4, 701 (1963).

[33] L. P. Gor'kov and G. M. Eliashberg, JETP 21, 940 (1965).

[34] B. L. Altshuler and B. I. Shklovskii, Zh. Eksp. Teor. Fiz. (Sov. Phys. JETP) 91 (64), 220 (127) (1986).

[35] B. I. Shklovskii, B. Shapiro, B. R. Sears, P. Lambrianides and H. B. Shore, Phys. Rev. B 47, 11487 (1993).

[36] R. Berkovits, Europhys. Lett. 25, 681 (1994).

[37] R. Berkovits, Y. Avishai, J. of Phys:Cond. Matt. 8, 389 (1996).

[38] M. Pascaud, G. Montambaux, Ann. der Physik 7, 406 (1998).

[39] R. Berkovits and B. I. Shklovskii, J. Phys. Condens. Matter 11, 779 (1999).

[40] P. H. Song, and D. L. Shepelyansky, Phys. Rev. B 61, 15546 (2000).

[41] V. Oganesyan and D. A. Huse, Phys. Rev. B 75, 155111 (2007); V. Oganesyan, A. Pal, and D. A. Huse, ibid. 80, 115104 (2009).

[42] J. I. Latorre and A. Riera, J. Phys. A: Math. Theor. 42, 504002 (2009).

[43] For a review see: P. A. Lee and T. V. Ramakrishnan, Rev. Mod. Phys. 57, 287 (1985).

[44] R. A. Römer and M. Schreiber, Phys. Rev. Lett. 78, 515 (1997).

[45] G. E. Andrews, The Theory of Partitions (Cambridge University Press, Cambridge) 1998, p. 1.

[46] G. H. Hardy, and S. Ramanujan, Proc. London Math. Soc. 17, 75, (1918).

[47] S. R. White, Phys. Rev. Lett. 69, 2863 (1992); Phys. Rev. B 48, 10345 (1993).

[48] U. Schollwöck, Rev. Mod. Phys. 77, 259 (2005); K. A. Hallberg, Adv. Phys. 55, 477 (2006). 